\title{
Revision tracheobronchoplasty: case report
}

\author{
Ammara A. Watkins, Jennifer L. Wilson, Mihir Parikh, Adnan Majid, Sidhu P. Gangadharan \\ Division of Thoracic Surgery and Interventional Pulmonology, Beth Israel Deaconess, Harvard Medical School, Boston, MA, USA \\ Correspondence to: Sidhu P. Gangadharan, MD. Chief, Division of Thoracic Surgery and Interventional Pulmonology, Beth Israel Deaconess Medical \\ Center, 185 Pilgrim Rd. W/DC 201 Boston, MA 02215, USA. Email: sgangadh@bidmc.harvard.edu.
}

\begin{abstract}
Tracheobronchoplasty, or posterior splinting of the airway with mesh, is a durable solution for patients with severe tracheobronchomalacia (TBM). Recurrent symptoms of TBM following tracheobronchoplasty are uncommon; however, when they occur can have significant impact on quality of life. Appropriate management of recurrent TBM requires a systematic and multidisciplinary collaborative approach. We present a patient with postoperative symptom recurrence requiring revisional tracheobronchoplasty to highlight the complexity of the disease's presentation, workup and treatment.
\end{abstract}

Keywords: Reoperative; revision; tracheobronchoplasty; tracheobronchomalacia (TBM); case report

Received: 06 October 2019; Accepted: 18 December 2019; Published: 25 November 2020.

doi: $10.21037 /$ ccts.2019.12.14

View this article at: http://dx.doi.org/10.21037/ccts.2019.12.14

\section{Introduction}

Tracheobronchomalacia is an increasingly recognized abnormality of the central airway that can cause dyspnea, cough, recurrent respiratory infections and respiratory insufficiency $(1,2)$. The hallmark of the disease is expiratory central airway collapse of at least $70 \%$ due to increased compliance of the membranous trachea (1-3). Surgical correction of malacic airways via posterior membranous stabilization with mesh, or tracheobronchoplasty, is a durable option for patients with severe disease, with good outcomes reported out to a median follow-up of six years (4). Nevertheless, recurrent symptoms requiring revisional surgery may occur $(2,5)$. The workup and subsequent operative approach amongst patients requiring revisional surgery are not described. We present such a case, where a patient had recurrent symptoms following tracheobronchoplasty and required revisional surgery.

\section{Case presentation}

A 49-year old woman with diabetes, gastroesophageal reflux disease (GERD), long-standing asthma and severe tracheobronchomalacia (TBM) underwent tracheobronchoplasty with Alloderm ${ }^{\mathrm{TM}}$ mesh via a right posterolateral thoracotomy at an outside institution.
Postoperatively she improved, but then about a year after her tracheobronchoplasty she reported recurrent wheezing, cough and shortness of breath. By four years following her operation, the progressive symptoms considerably impacted her quality of life. She was unable to walk 2 blocks without shortness of breath and had been admitted at least six times in the past year due to respiratory distress. Pulmonary function tests are presented in Table 1. Due to the potential multifactorial nature of her symptoms, initially our approach was to focus on maximal medical management. Her proton pump inhibitor was increased should there have been any contribution from her GERD. Her bronchodilator medications were also adjusted. She was also given a trial of pulse steroids and participated in pulmonary rehabilitation to medically optimize treatment of her asthma. Unfortunately, none of these medical interventions improved her symptoms. By seven years out from her tracheobronchoplasty, dynamic CT of the trachea demonstrated $65 \%$ cross-sectional airway luminal collapse during exhalation and her bronchi had nearly $100 \%$ collapse (Figure 1A). This was progressive from her initial CT at year five. Dynamic flexible bronchoscopy also revealed severe bilateral bronchomalacia to the distal segments and nearly $100 \%$ collapse during passive exhalation (Figure 1B). She underwent a short-term stent trial and had improvement in her symptoms and pulmonary function tests (Table 1) with 
Table 1 Preoperative, post- stent and postoperative pulmonary function tests

\begin{tabular}{lccr} 
& Preoperative & Post- stent placement & Postoperative \\
\hline FVC $^{*}$ & 59 & 69 & 76 \\
FEV1 $^{*}$ & 47 & 55 & 61 \\
FEV1/FVC* $^{*}$ & 79 & 79 & 30 \\
Six-minute walk test & 234.6 meters & 371 meters & 344 meters \\
\hline
\end{tabular}

*, percent predicted. FVC, forced vital capacity; FEV1, forced expiratory volume in the 1st second.
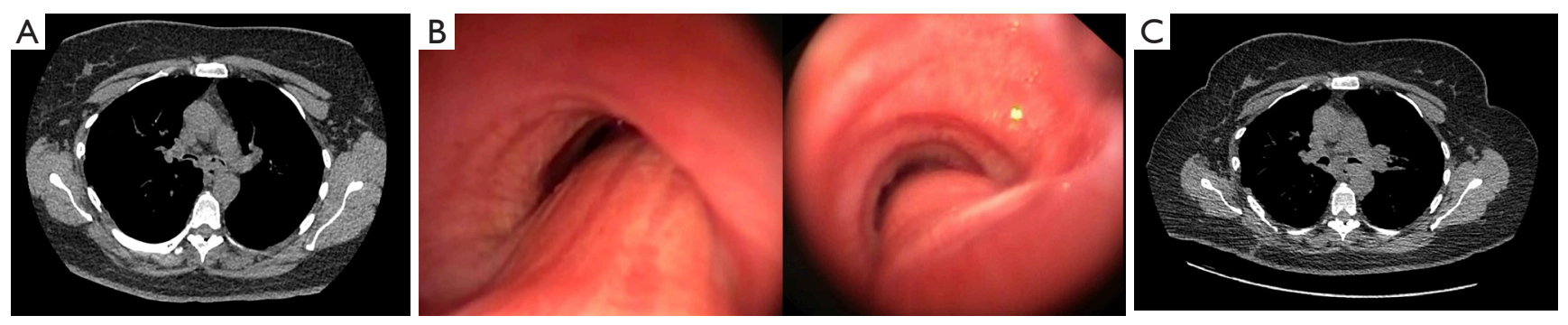

Figure 1 Preoperative CT (A) and bronchoscopic (B) findings demonstrating airway collapse in the right and left mainstem bronchi, postoperative CT shows improvement (C).

the stent in place. Upon removal of the stent she had return of symptoms. We therefore felt she would benefit from revisional tracheobronchoplasty.

The operation was done through her previous posterolateral thoracotomy with the patient in left lateral decubitus position utilizing a single lumen tube and right bronchial blocker. An epidural was placed preoperatively. There were significant adhesions to the posterior aspect of the trachea. The esophagus was carefully dissected off the trachea with preservation of the vagus nerve. Sutures were cut off the prior mesh as they were encountered. The mesh was then carefully removed from the trachea. Interestingly, the acellular dermal mesh was itself minimally adherent with the trachea. A custom Y-shaped polypropylene mesh, which is our institutional preference for splinting material, was then fashioned. The mesh was then secured with four rows of polypropylene sutures to the distal trachea, and bilateral bronchi as described previously by our group $(1,2,6)$. Simultaneous bronchoscopy was used to insure partial-thickness suture placement. A small-bore chest tube was placed, and the patient was transferred to intensive care unit.

Despite the arduous seven-hour reoperation, her postoperative course was unremarkable. Most recently she was seen in clinic four months following her revisional tracheobronchoplasty without any recurrent symptoms, and her pulmonary function tests, six-minute walk test, and collapsibility indices on CT were all improved (Table 1, Figure 1C).

All procedures performed in this study were in accordance with the ethical standards of the institutional and/or national research committee(s) and with the Helsinki Declaration (as revised in 2013). Written informed consent was obtained from the patient for publication of this case report and any accompanying images. A copy of the written consent is available for review by the editorial office of this journal.

\section{Discussion}

Although uncommon amongst the general population, TBM is frequently seen diagnosed in patients with asthma, GERD and other respiratory conditions, often after considerable delay $(2,7)$. This patient's comorbidities and her history of a prior tracheobronchoplasty made identification of the etiology of her debilitating symptoms challenging. Approaching her workup systematically as we do in all patients under consideration for tracheobronchoplasty was particularly important given increased difficulty and risks of revisional surgery. It was necessary to medically optimize her asthma and GERD as much as possible because these conditions may mimic symptoms of TBM (2). Dynamic CT 
and bronchoscopy remain the gold standard in delineating the extent and morphology of airway collapse $(2,8)$.

Our team also utilizes a stent trial to gauge likelihood of benefit from tracheobronchoplasty once a patient is considered a candidate for possible surgical repair (9). Deployment of the stent(s) in the thoracic trachea and bilateral mainstem bronchi mimics the anatomic areas that would be stabilized with a tracheobronchoplasty. Tracheobronchoplasty appears to be the most durable solution for patients with severe TBM. Current work in our unit is ongoing to identify factors that predict the risk for recurrent TBM following tracheobronchoplasty, which while rare, can be extremely impactful on patients' quality of life. In this patient, we suspected that lack of incorporation of the acellular dermis mesh may have contributed to recurrent collapse. It is speculative, but perhaps polypropylene mesh may promote a stronger inflammatory reaction and tissue ingrowth, and, thus, durability of posterior splinting. No side-by-side comparisons of mesh choice in tracheobronchoplasty are available and the polypropylene mesh use also has its side effects, such as mesh erosion into the airway $(2,10)$.

Reoperative tracheobronchoplasty does require some modification in technique but the surgical principals are the same. It is important to work on the posterior trachea and avoid lateral dissection that may devascularize the subsequent repair particularly with difficult tissue planes. Although not necessary for this case, some portions of the membranous wall can become too thin after mesh removal to accommodate partial thickness sutures for the second repair. At times when this is the case, we use absorbable sutures to anchor the mesh in case full-thickness passes are required to achieve suitable purchase. We perform all suture placements under bronchoscopic visualization. We also use a leak test under sterile water should there be any tears in the posterior membrane. Occasionally repair stitches are required in the trachea and these are buttressed with autologous pleura or pericardial pledgets to facilitate repair. Closure of the defect is confirmed with bronchoscopy as with all stitches placed into the mesh. After mesh placement, real-time bronchoscopy also allows us to visualize any remaining malacic areas that require reinforcement sutures into the mesh to optimize tension.

Despite the complex nature of revisional surgery, there is often minimal alteration to our institution's enhanced recovery after surgery (ERAS) pathway for tracheobronchoplasty. All patients have a preoperative epidural placed to optimize pain control. Postoperatively patients are monitored in the ICU for 48 hours. Most patients are extubated postoperatively; however, if there is any concern particularly given the longer operative time in revisional tracheobronchoplasty, they are left intubated the day of surgery. Pulmonary optimization with chest physical therapy, nebulizers and early mobilization is key. Diet is advanced when there is no longer concern for aspiration, and the chest tube is removed once drainage diminishes. While this case report is limited by its small sample size, we plan to analyze long-term data as more patients requiring revisional tracheobronchoplasty are accrued.

\section{Conclusions}

Recurrent tracheobronchomalacia following tracheobronchoplasty is a rare occurrence, but likely to increase in frequency as more awareness and surgical treatment for tracheobronchomalacia is offered. It is important to be systematic in the workup of a patient under consideration for revision tracheobronchoplasty and adhere to the surgical principles one would utilize in an index operation.

\section{Acknowledgments}

Funding: None.

\section{Footnote}

Conflicts of Interest: All authors have completed the ICMJE uniform disclosure form (available at https://ccts. amegroups.com/article/view/10.21037/ccts.2019.12.14/ coif). The authors have no conflicts of interest to declare.

Ethical Statement: The authors are accountable for all aspects of the work in ensuring that questions related to the accuracy or integrity of any part of the work are appropriately investigated and resolved. All procedures performed in this study were in accordance with the ethical standards of the institutional and/or national research committee(s) and with the Helsinki Declaration (as revised in 2013). Written informed consent was obtained from the patient for publication of this case report and any accompanying images. A copy of the written consent is available for review by the editorial office of this journal.

Open Access Statement: This is an Open Access article distributed in accordance with the Creative Commons Attribution-NonCommercial-NoDerivs 4.0 International 
License (CC BY-NC-ND 4.0), which permits the noncommercial replication and distribution of the article with the strict proviso that no changes or edits are made and the original work is properly cited (including links to both the formal publication through the relevant DOI and the license). See: https://creativecommons.org/licenses/by-nc-nd/4.0/.

\section{References}

1. Gangadharan SP. Tracheobronchomalacia in adults. Semin Thorac Cardiovasc Surg 2010;22:165-73.

2. Buitrago DH, Wilson JL, Parikh M, et al. Current concepts in severe adult tracheobronchomalacia: evaluation and treatment. J Thorac Dis 2017;9:E57-66.

3. Litmanovich D, O'Donnell CR, Bankier AA, et al. Bronchial collapsibility at forced expiration in healthy volunteers: assessment with multidetector CT. Radiology 2010;257:560-7.

4. Bezuidenhout AF, Boiselle PM, Heidinger BH, et al. Longitudinal Follow-up of Patients With Tracheobronchomalacia After Undergoing Tracheobronchoplasty: Computed Tomography Findings

doi: $10.21037 /$ ccts.2019.12.14

Cite this article as: Watkins AA, Wilson JL, Parikh M, Majid A, Gangadharan SP. Revision tracheobronchoplasty: case report. Curr Chall Thorac Surg 2020;2:40. and Clinical Correlation. J Thorac Imaging 2019;34:278-83.

5. Alape DE, Gangadharan S, Folch E, et al.

Tracheobronchoplasty for severe tracheobronchomalacia: Short and Long-term Outcomes. Am J Respr Crit Care Med 2016;193:A3386.

6. Gangadharan SP, Bakhos CT, Majid A, et al. Technical aspects and outcomes of tracheobronchoplasty for severe tracheobronchomalacia. Ann Thorac Surg 2011;91:157480; discussion 1580-1.

7. Dal Negro RW, Tognella S, Guerriero M, et al. Prevalence of tracheobronchomalacia and excessive dynamic airway collapse in bronchial asthma of different severity. Multidiscip Respir Med 2013;8:32.

8. Majid A, Gaurav K, Sanchez JM, et al. Evaluation of tracheobronchomalacia by dynamic flexible bronchoscopy. A pilot study. Ann Am Thorac Soc 2014;11:951-5.

9. Ernst A, Majid A, Feller-Kopman D, et al. Airway stabilization with silicone stents for treating adult tracheobronchomalacia: a prospective observational study. Chest 2007;132:609-16.

10. Wright CD, Mathisen DJ. Tracheobronchoplasty for tracheomalacia. Ann Cardiothorac Surg 2018;7:261-5. 